\title{
ON SCALING OF SCHRÖDINGER EQUATION AND SOME RE- SULTS FOR HEAVY QUARKS MESONS
}

\author{
NICOLA FABIANO $^{1, \star}$, STOJAN RADENOVIĆ ${ }^{2}$ \\ 1 "Vinča" Institute of Nuclear Sciences - National Institute of the Republic of Serbia, University of Belgrade, Mike Petrovića \\ Alasa 12-14, 11351 Belgrade, Serbia \\ ${ }^{2}$ Faculty of Mechanical Engineering, University of Belgrade, Kraljice Marije 16, 11120 Beograd 35, Serbia
}

\begin{abstract}
We consider the scaling of the Schrödinger equation in order to explicitly compute the energy density levels for a specific class of potentials. The resulting eigenvalues spectrum is compared to the heavy quarks mesons spectroscopy, showing a fair agreement with experimental data for the $J / \psi$ and, for the heavier case $\Upsilon$, an excellent agreement with the experimental data.
\end{abstract}

Keywords: Schrödinger equation, Semiclassical methods, Heavy quarks.

\section{DETERMINATION OF THE POTENTIAL ENERGY FROM THE PERIOD OF OSCILLATION}

In this paper we will consider the Schrödinger equation with a class of power law potential

$$
H=\frac{p^{2}}{2 m}+A x^{\gamma}=-\hbar^{2}\left(\frac{d}{d x}\right)^{2}+A x^{\gamma},
$$

( $A$ is a constant) whose energy eigenvalues will be compared to the ones of heavy quark mesons. Consider the energy conservation equation

$$
E=\frac{m \dot{x}^{2}}{2}+V(x)
$$

and solve for $\dot{x}$ :

$$
\dot{x}=\left[\sqrt{\frac{2(E-V(x))}{m}}\right] .
$$

In order to obtain the period of motion we solve for $d t / d x$ :

$$
\begin{array}{r}
\int_{0}^{T(E)} d t=T(E)=4\left(\frac{m}{2}\right)^{1 / 2} \int_{0}^{x(E)} \frac{1}{\sqrt{E-V(x)}} d x= \\
2(2 m)^{1 / 2} \int_{V(0)=0}^{E} \frac{(d x / d V)}{\sqrt{E-V}} d V,
\end{array}
$$

here $x=x(V)$ is the inverse function of the potential and we assume for simplicity that $V(0)=0$. In order to write explicitly this integral, divide the equation by $\sqrt{\alpha-E}$ where $\alpha$ is a parameter such that

$$
0 \leq V \leq E \leq \alpha
$$

and integrate first over the energy $\int_{0}^{\alpha} d E$ swapping integration order (Landau \& Lifshitz, 1960):

$$
\begin{array}{r}
\int_{0}^{\alpha} \frac{T(E)}{\sqrt{\alpha-E}} d E= \\
\int_{0}^{\alpha} d V(d x / d V) \int_{V}^{\alpha} d E[(E-V)(\alpha-E)]^{-1 / 2} .
\end{array}
$$

Last integral of eq. (3) in $d E$ yields:

$$
\int_{V}^{\alpha} d E[(E-V)(\alpha-E)]^{-1 / 2}=-2 \arcsin (-1)=\pi
$$

for a final result of

$$
2 \pi(2 m)^{1 / 2} x(\alpha)
$$

Replacing $\alpha$ with $V$ we obtain an expression for the shape of the potential in terms of the period

$$
x(V)=\frac{1}{2 \pi(2 m)^{1 / 2}} \int_{0}^{V} \frac{T(E)}{\sqrt{V-E}} d E .
$$

For example, if the period $T$ is independent of energy, then

$$
x(V)=C \int_{0}^{V} \frac{1}{\sqrt{V-E}} d E=2 C \sqrt{V}
$$

where $C$ is a constant. Inverting the above relation we recover the usual harmonic oscillator

$$
V(x)=A x^{2}
$$

\section{SEMICLASSICAL APPROACH TO QUANTUM ENERGY DENSITY LEVELS}

A similar method achieves results in semiclassical approximation WKB of a symmetric monotonic potential in one dimension. Starting from the Bohr-Sommerfeld quantisation condition

$$
\frac{1}{2 \pi} \oint p d x=\left(n+\frac{1}{2}\right) \hbar, \text { for } n=0,1,2, \ldots
$$

noticing that $\oint p d x=2 \int_{a}^{b} p d x$, where $a$ and $b$ are the turning points which solve the equation $E-V(x)=0$, and working with the units where $\hbar=1$ one has

$$
\int_{0}^{x_{0}} d x\left[2 m\left(E_{n}-V(x)\right)\right]^{1 / 2}=\left(n+\frac{1}{2}\right) \pi
$$


$x_{0}$ being the classical inversion point $V\left(x_{0}\right)=E, V(0)=0$, and differentiating both sides with respect to $n$ we have

$$
\frac{1}{2} \int_{0}^{x_{0}}\left[\frac{2 m}{E-V}\right]^{1 / 2}\left(\frac{\partial E}{\partial n}\right) d x=\pi,
$$

that could be rewritten as

$$
\int_{V(0)=0}^{E} d V(d x / d V)\left[\frac{2 m}{E-V}\right]^{1 / 2}=\frac{2 \pi}{\partial E / \partial n}
$$

which in form is quite similar to eq. (2), and we will proceed as before. Inserting a term $\int_{0}^{\alpha} d E(\alpha-E)^{-1 / 2}$ and interchanging the order of integration we find

$$
\begin{array}{r}
\int_{0}^{\alpha} d V(d x / d V) \int_{V}^{\alpha} \frac{1}{(\alpha-E)^{1 / 2}(E-V)^{1 / 2}} d E= \\
2 \pi \int_{0}^{\alpha} \frac{1}{[2 m(\alpha-E)]^{1 / 2}(\partial E / \partial n)} d E .
\end{array}
$$

The integral in $d E$ on lhs has already been encountered in (3) and its value is $\pi$. Upon renaming $\alpha \rightarrow V$ we are left with the result

$$
\begin{array}{r}
x(V)=2 \int_{0}^{V} \frac{1}{[2 m(V-E)]^{1 / 2}(\partial E / \partial n)} d E= \\
2 \int_{0}^{V} \frac{(\partial n / \partial E)}{[2 m(V-E)]^{1 / 2}} d E
\end{array}
$$

where $(\partial n / \partial E)$ is the density of eigenvalues function.

Consider for instance a constant level density, i.e. where $(\partial E / \partial n)$ is constant. Then the above integral furnishes us with the result

$$
x(V)=A^{\prime} \sqrt{V}
$$

where $A^{\prime}$ is some constant. Inverting the relation we obtain the harmonic oscillator as expected

$$
V(x)=A x^{2} .
$$

\section{SOME NOTABLE RESULTS}

The results obtained so far are valid in one dimensional quantum mechanics, therefore they also hold for symmetric central potentials for the radial part. We should recall some notable results for integrable systems.

\section{Coulombic potential}

$$
\begin{gathered}
V(x)=-\frac{A}{x} \\
E_{n}=-\frac{R}{n^{2}} \text { implies } n(E) \sim E^{-1 / 2}
\end{gathered}
$$

Harmonic oscillator

$$
\begin{gathered}
V(x)=A x^{2} \\
E_{n}=\left(n+\frac{1}{2}\right) \hbar \omega \text { implies } n(E) \sim E
\end{gathered}
$$

Infinite square well

$$
\begin{gathered}
V(x)=0 \text { if } 0<x<L, \text { else }+\infty \\
E_{n}=C n^{2} \text { implies } n(E) \sim E^{1 / 2}
\end{gathered}
$$

\section{General results}

Those potentials have been listed for energy $E$ ordered as increasing function of $n$.

With the knowledge of density function and main equation (7) we could infer some general results. First, with the change of variable

$$
\xi=\frac{E}{V}
$$

and considering the Jacobian, we are left with

$$
x(V)=C \sqrt{V} \int_{0}^{1} d \xi(1-\xi)^{-1 / 2}\left[\frac{\partial n(\xi V)}{\partial E}\right]
$$

$C$ being a constant. Now when the density function has some homogenity properties one could infer some actual results. With the power law Ansatz

$$
\frac{\partial n(E)}{\partial E} \sim E^{a}
$$

that is

$$
n(E) \sim E^{a+1}
$$

and inverting the relation one obtains

$$
\begin{aligned}
& E(n) \sim n^{\left(\frac{1}{a+1}\right)}, \\
& \frac{\partial E(n)}{\partial n} \sim n^{\left(-\frac{a}{a+1}\right)}
\end{aligned}
$$

for $a \neq-1$, from eq. (8) we obtain

$$
x(V)=C V^{\left(a+\frac{1}{2}\right)} \int_{0}^{1} d \xi(1-\xi)^{-1 / 2} \xi^{a}=C V^{\left(a+\frac{1}{2}\right)} \beta\left(\frac{1}{2}, a+1\right),
$$

which leads to

$$
x(V) \sim V^{\left(a+\frac{1}{2}\right)}
$$

and inverting the equation we obtain the potential as a function of $x$

$$
V(x) \sim x^{\left(\frac{2}{2 a+1}\right)} .
$$

Being a semiclassic approximation, the larger $n$ the better agreement with the exact result is obtained.

\section{A few examples}

- For a linear potential:

$$
\begin{gathered}
V(x) \sim x \text { implies } a=\frac{1}{2} \\
n(E) \sim E^{(3 / 2)} ; \frac{\partial n(E)}{\partial E} \sim E^{(1 / 2)} ; E(n) \sim n^{(2 / 3)} .
\end{gathered}
$$

- For an anharmonic potential:

$$
V(x) \sim x^{4} \text { implies } a=-\frac{1}{4}
$$




$$
n(E) \sim E^{(3 / 4)} ; \frac{\partial n(E)}{\partial E} \sim E^{(-1 / 4)} ; E(n) \sim n^{(4 / 3)} .
$$

- For $E \sim 1 / n$ :

$$
\begin{gathered}
E(n) \sim \frac{1}{n} \text { implies } a=-2 \\
V(x) \sim x^{(-2 / 3)} \\
n(E) \sim E^{(-1)} ; \frac{\partial n(E)}{\partial E} \sim E^{(-2)} ; E(n) \sim n^{(-1)}
\end{gathered}
$$

less singular in the origin than the Coulombic case $1 / x$.

- Suppose we search a potential for which the energy density increases slightly with the energy, (that is indistinguishable from a logarithm) i.e.

$$
\frac{\partial n(E)}{\partial E} \sim E^{\varepsilon}
$$

where $\varepsilon>0$ is small (the analog case with slight decrease is obtained for $\varepsilon<0)$. Then we obtain

$$
\begin{gathered}
V(x) \sim x^{\left(\frac{2}{1+2 \varepsilon}\right)} \approx x^{2(1-2 \varepsilon)} \\
n(E) \sim E^{(1+\varepsilon)} ; \frac{\partial n(E)}{\partial E} \sim E^{\varepsilon} ; E(n) \sim n^{\left(\frac{1}{1+\varepsilon}\right)} \approx n^{(1-\varepsilon)} .
\end{gathered}
$$

- Observe that for the case of an infinite square well where $E \sim n^{2}$ the power law potential resulting from (11) has a singularity.

In table 1 . we present the scaling results obtained for some power law potentials.

Table 1. Scaling for different potentials, ranging from anharmonic $x^{4}$ to Coulombic potential $1 / x$.

\begin{tabular}{||c|c|c|c||}
\hline Potential & $E(n) \sim n^{1 /(a+1)}$ & $\partial E / \partial n$ & $a$ \\
\hline$x^{4}$ & $n^{(4 / 3)}$ & $n^{(1 / 3)}$ & $-1 / 4$ \\
\hline$x^{2}$ & $n^{1}$ & const & 0 \\
\hline$x^{1}$ & $n^{(2 / 3)}$ & $n^{(-1 / 3)}$ & $+1 / 2$ \\
\hline$x^{-1}$ & $n^{(-2)}$ & $n^{(-3)}$ & $-3 / 2$ \\
\hline
\end{tabular}

\section{BEYOND LEADING ORDER}

The quantisation condition met in (4) is only the first order approximation of the whole WKB procedure. To summarize the method, consider the eigenvalues problem of the Schrödinger equation

$$
\hbar^{2} y^{\prime \prime}(x)=2 m[V(x)-E] y(x)=-p^{2}(x) y(x)
$$

with the wavefunctions $y(x)$ obeying the boundary conditions $y( \pm \infty)=0$. For the WKB procedure we will consider $\hbar$ as an expansion parameter, eventually letting its value to $\hbar \rightarrow 1$ (Bender et al., 1977). The series expansion for the wavefunction is given by

$$
y(x)=\exp \left[\frac{1}{\hbar} \sum_{k=0}^{+\infty} \hbar^{k} S_{k}(x)\right],
$$

plugging back (13) in (12) and comparing the coefficients of the same power of $\hbar$ one obtains the equation

$$
S_{0}^{\prime}(x)=\left[-p^{2}(x)\right]^{1 / 2}
$$

together with the recursive relation valid for any order of $S_{k}(x)$ :

$$
2 S_{0}^{\prime} S_{k}^{\prime}+\sum_{j=1}^{k-1} S_{j}^{\prime} S_{j-k}^{\prime}+S_{k-1}^{\prime \prime}=0, \text { for } k \geq 0
$$

The first computed terms up to $O\left(\hbar^{3}\right)$ are given by

$$
\begin{array}{r}
S_{1}^{\prime}=-\frac{1}{2} \frac{p^{\prime}}{p}=-\frac{1}{2}(\ln p)^{\prime}, \\
S_{2}^{\prime}=-\frac{i}{8 p^{3}}\left[2 p p^{(2)}+3\left(p^{\prime}\right)^{2}\right], \\
S_{3}^{\prime}=\frac{1}{16 p^{6}}\left[2 p^{3} p^{(4)}-16 p^{2} p^{\prime} p^{(3)}-10 p^{2}\left(p^{(2)}\right)^{2}+\right. \\
\left.67 p\left(p^{\prime}\right)^{2} p^{(2)}-45\left(p^{\prime}\right)^{4}\right] .
\end{array}
$$

Once solved for $S_{k}^{\prime}(x)$ one obtains a generalization of the formulæ (4),(5) to all orders of WKB expansion:

$$
\frac{1}{2 i} \oint \sum_{k=0}^{+\infty} S_{k}(x)^{\prime} d x=n \pi .
$$

For the energy eigenvalues of the power law potential (1) one obtains the following result (Parisi G. in: Chudnovsky \& Chudnovsky, 2006) in the limit $n \rightarrow+\infty$

$$
E_{n}=n^{2 \gamma /(\gamma+2)}\left[C_{0}+\frac{C_{1}}{n^{2}}+O\left(\frac{1}{n^{4}}\right)\right],
$$

where $C_{0}, C_{1}$ are constants. Observe that the first term coincides with the results obtained in Section (Some Notable Results).

\section{RELATION TO HEAVY MESONS}

In order to verify the results obtained so far, we will make use of a comparison to the values of some heavy meson states. It is well known that (Fabiano, 1998) heavy mesons, i.e. $q \bar{q}$ states can be well described by an effective radial potential $V(r)$ in a Schrödinger equation

$$
H \psi=\left[-\frac{\nabla^{2}}{2 \mu}+V(r)\right] \psi=E \psi,
$$

$\mu$ being the reduced mass of the state, mimicking the QCD phenomenology, incorporating short distance behaviour, that is approaching a Coulombic potential for $r \rightarrow 0$, and long distance behaviour, approaching a linear nonperturbative term due to QCD confinement for large values of $r$. The heavier the meson, the closer the $q \bar{q}$ pair orbits together, and is better described by a semiclassical picture. Moreover, the relativistic effects are less relevant in those heavier cases (Fabiano, 2001).

The mass of an $n S$ quark bound state is given by the expression (Fabiano, 1998)

$$
M(n)=2 m+E(n)
$$

$M(n)$ being the mass of the $n$ level bound state, $m$ the quark mass and $E(n)$ the binding energy of the state. We will make use of the results obtained in Section (Some Notable Results) for a power 
law potential and make a fit to $J / \psi$ and $\Upsilon n S$ states and their excitations as a function of $n=1 \ldots 4$, with data provided in (Particle Data Group et al., 2020; Barnes, 2006). The power law potential will average between the two behaviours depicted above, for small and large values of the quark-antiquark distance $r$, providing a kind of "effective potential". Our aim is not to introduce another power law potential (see (Fabiano, 1998) and references therein for a partial list), but rather to verify the validity of the WKB procedure and its accuracy within the approximation order considered and when $n$ is not going to infinity.

Beginning with $J / \psi$ state we have the following results for the mass of its excited levels $n S$ as a function of the quantum number $n$, together with the first order approximation fit obtained for a power law potential presented in fig. 1 .

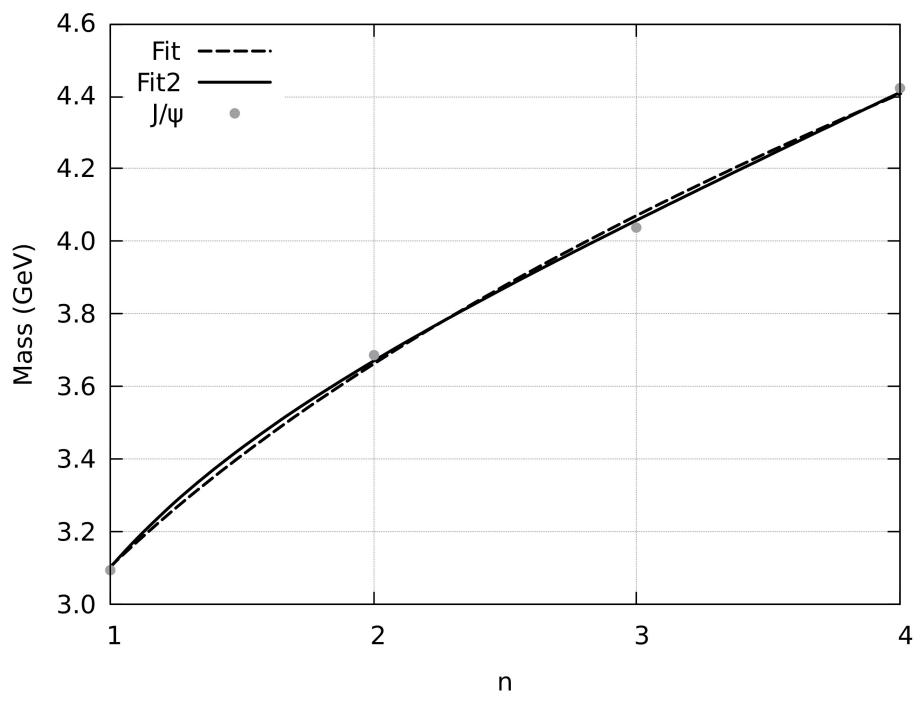

Figure 1. $J / \psi n S$ states mass and fit of a power law potential as a function of $n$, for first (Fit) and second order energy spectrum (Fit2)

The resulting energy scaling and potential from the $J / \psi$ fit is given by

$$
E(n) \sim n^{0.413} \text { yields } V(r) \sim r^{0.520} .
$$

The other fit parameters from equations (20) and (22) are $m=$ 0.706, $\gamma=0.520, C_{0}=1.690$.

Comparing the results obtained for the $J / \psi$ excited states in fig. 1. we can see that the second order approximation gives a more accurate fit than the first order one, as expected. The second order approximation curve is even closer to the experimental values, almost passing through all data points. The resulting energy scaling and potential from the $J / \psi$ fit with a second order approximation is given by

$$
E(n) \sim n^{1.398} \text { yields } V(r) \sim r^{4.643}
$$

which is rather different from the previous results of the first order, showing that to achieve a better accuracy for the $J / \psi$ states effective potential some relativistic effects should be considered as well. The other fit parameters are $m=2.058, \gamma=4.643, C_{0}=$ $0.113, C_{1}=-1.130$. Because of its relatively low mass the $J / \psi$ state is not completely a "nonrelativistic state" (Fabiano, 1998, 2001) as discussed above.

Concerning the semiclassical approximation, as there are four experimental points, it is not possible to proceed further in the approximation order of (20) as there would be more variables than data points, leaving some of them with arbitrary values, leading to multiple (maybe infinitely many) solutions that fit the data equally well, that is a so called "underdetermined" problem. Another remark is that as the fit for equation (22) is not given by a polynomial function, even if there are an equal number of variables and points, there is no guarantee that the fit will pass exactly though all those points.

For the $\Upsilon$ state and its $n S$ excitations, and the fit for a power law potential we obtain the results shown in fig. 2 .

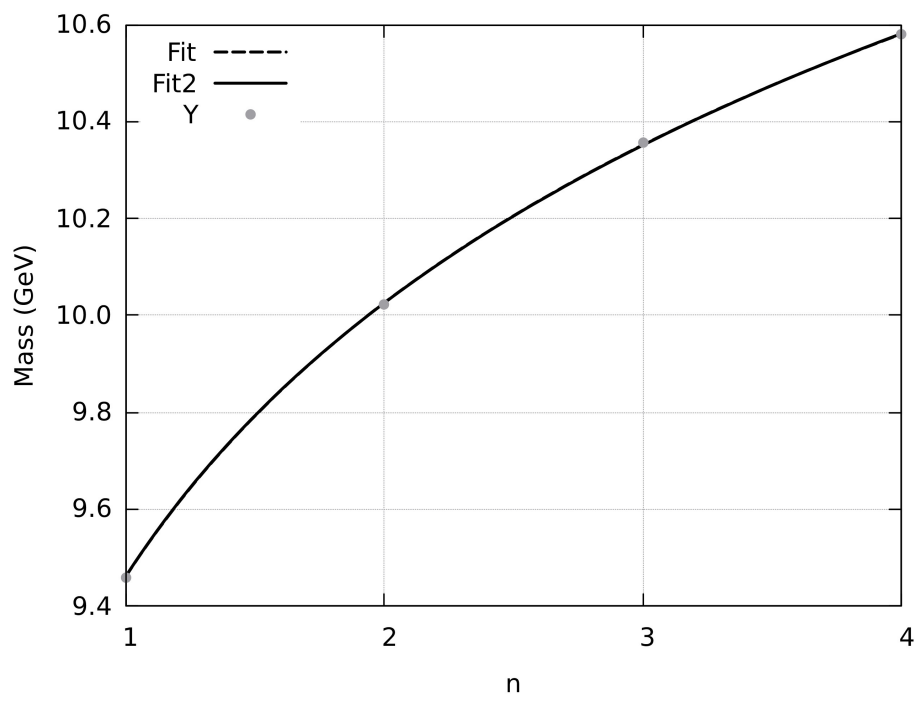

Figure 2. $\Upsilon n S$ states mass and fit of a power law potential as a function of $n$, for first (Fit) and second order energy spectrum (Fit2)

The resulting energy scaling and effective potential from the $\Upsilon$ fit at first order approximation is given by

$$
E(n) \sim n^{0.011} \text { yields } V(r) \sim r^{0.011} .
$$

The other fit parameters from equations (20) and (22) are $m=$ $-33.448, \gamma=0.011, C_{0}=76.359$.

The comparison of the $\Upsilon$ excited states in fig. 2. shows a different scenario with respect to the $J / \psi$ one of fig. $1 .:$ the result of first order approximation is already a very good one, as it completely overlaps with the experimental points. The second order approximation is virtually indistinguishable from the first one. The resulting energy scaling and potential from the $\Upsilon$ fit with a second order approximation is given by

$$
E(n) \sim n^{0.017} \text { yields } V(r) \sim r^{0.017},
$$

which is quite close to the one obtained by the first order approximation. The other fit parameters are $m=-18.159, \gamma=$ 
0.017, $C_{0}=45.807, C_{1}=-0.028$. Therefore, the $\Upsilon$ states actually behave like "nonrelativistic semiclassical states", providing with an excellent agreement with the energy levels and a good approximation for the purely power law potential.

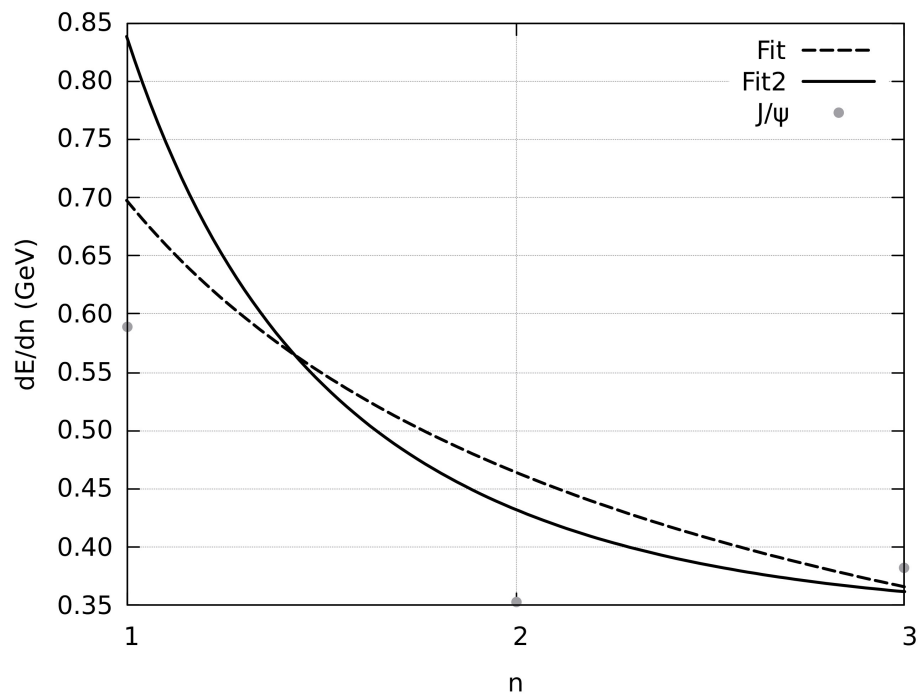

Figure 3. Derivative of $\mathrm{J} / \psi n S$ energy levels with respect to $n$ and fit of a power law potential as a function of $n$, for first (Fit) and second order energy spectrum (Fit2)

We therefore evince from figs 1 . and 2. that there is a good agreement with the $J / \psi$ data and an excellent agreement with the $\Upsilon$ states, and the latter behaves, as expected because much heavier, more like a nonrelativistic semiclassical system. The lower exponent of the power law potential in the latter case is also to be expected, as could be seen in table 1 .

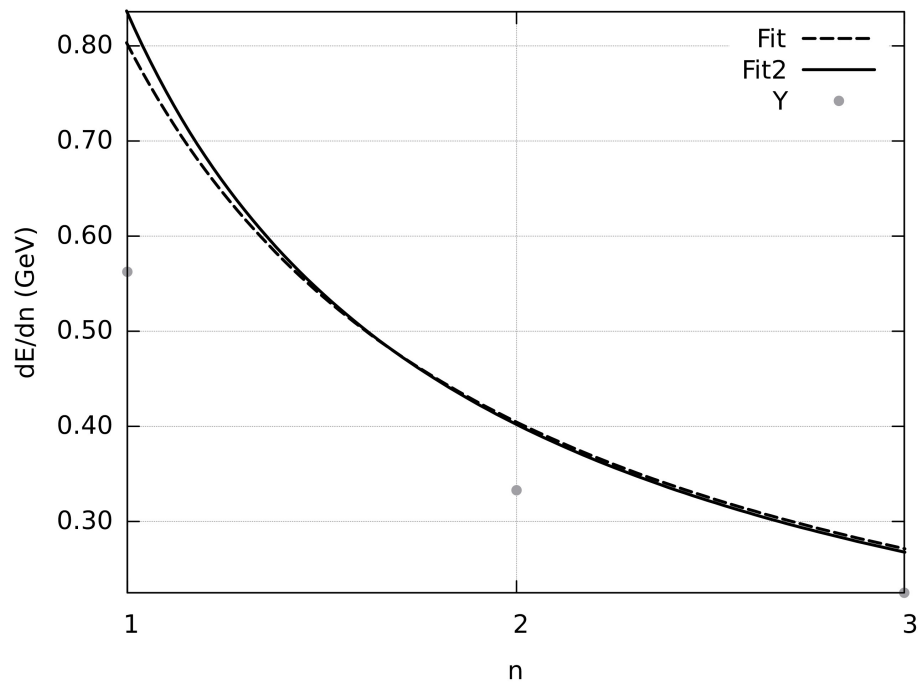

Figure 4. Derivative of $\Upsilon n S$ energy levels with respect to $n$ and fit of a power law potential as a function of $n$, for first (Fit) and second order energy spectrum (Fit2)

Then we consider the derivative of the density of states $\partial E / \partial n$. The data for $n S$ excited states of $J / \psi$ and $\Upsilon$ can not provide us with a proper derivative, but rather with a discrete approxima- tion of energy differences divided by unitary step, $\Delta n=1$. This should suffice for our analysis, where we compare the results obtained from the experimental data to the fitted curve of eq. (22) once taken the derivative with respect to $n$.

Starting from the $J / \psi$ excitations from fig. 3. one could observe that, even if the two fits do not meet the experimental points (as expected because they are not proper derivatives) they both reproduce the behaviour of the points as a function of $n$, with the second order approximation being closer to the data.

For the $\Upsilon$ case the same reasoning of the $J / \psi$ case is valid. This time however there is almost not difference between first and second order approximation as expected, and the agreement with the experimental points is also better fig. 4 .

\section{CONCLUSIONS}

We have considered the behaviour of the energy spectrum of a Schrödinger equation with a particular class of potential. The results for the energy levels have been obtained with the aid of the WKB technique. The spectrum has been compared to the heavy meson $n S$ states of $J / \psi$ and $\Upsilon$ providing, at least for the energy levels, respectively a very good and an excellent agreement with data, even though the quantum number $n$ does not tend to infinity and is not very high either. We have checked the validity of the WKB results used even when not employing a high approximation order. Those results prove once more the validity and the accuracy of the Schrödinger equation approach for a phenomenological description of some QCD phenomena.

\section{REFERENCES}

Barnes, T. 2006, The XYZs of charmonium at BES, International Journal of Modern Physics A, 21(27), pp. 5583-5591. https://doi.org/10.1142/S0217751X0603477X

Bender, C. M., Olaussen, K., \& Wang, P. S. 1977, Numerological analysis of the WKB approximation in large order, Physical Review D, 16(6), pp. 1740.

Fabiano, N. 1998, Top Mesons, The European Physical Journal C-Particles and Fields, 2(2), pp. 345-350.

Fabiano, N. 2001, Estimates of threshold cross section for stoponium production at e+e- colliders, The European Physical Journal C-Particles and Fields, 19 (3), pp. 547-551. https://doi.org/10.1007/s100520100638

Landau, L. D. \& Lifshitz, E. M. 1960, Mechanics. Vol. 1 of: Course of Theoretical Physics, Pergamon Press Ltd.

Parisi G. in: Chudnovsky, D. \& Chudnovsky, G. 2006, The Riemann Problem, Complete Integrability and Arithmetic Applications: Proceedings of a Seminar Held at the Institut Des Hautes Etudes Scientifiques, Bures-sur-Yvette, France and at Columbia University, NY, USA, 925, pp. 1979-1980. Springer

Particle Data Group, Zyla, P. A., \& Barnett, R. M. 2020, Review of Particle Physics, Progress of Theoretical and Experimental Physics, 2020(8), https://doi.org/10.1093/ptep/ptaa104 